\title{
A miniaturized MID-IR-Spectrometer based on a linear variable filter and pyroelectric line array - Monitoring oil condition
}

\author{
Benjamin R. Wiesent ${ }^{1}$, Dipl.-Ing. Daniel G. Dorigo ${ }^{2}$, Prof. Dr.-Ing. habil. Dr. h.c. Alexander W. Koch ${ }^{2}$ \\ ${ }^{1}$ Technische Universität München, Arcisstr. 2180290 München, Germany \\ Benjamin.wiesent@comline-elektronik.de \\ ${ }^{2}$ Technische Universität München, Arcisstr. 2180290 München, Germany
}

\begin{abstract}
Miniaturized mid-IR spectrometers have an enormous potential to solve a plurality of different measurement problems. IR-Spectroscopy can be used for qualitative and quantitative measurements of chemical compounds and their concentrations in solid, gaseous and fluid phase. Building the IRspectrometer small, cost effective and robust without any moving parts enables an online or even inline measurement in process monitoring, health monitoring or quality control tasks.

In this paper we present a miniaturized MID-IR spectrometer without any moving parts that operates in the wavelength range from $5.5-11 \mu \mathrm{m}(1800-900 \mathrm{~cm}-1)$. The miniaturized spectrometer is based on a linear variable filter (LVF) as dispersive element mounted on top of a pyroelectric line sensor. The performance of this miniaturized low cost MID-IR spectrometer is demonstrated in an online oil condition monitoring application. Therefore a number of representative used oil samples at different stages of degradation is measured and compared to a FTIR based complimentary analysis.
\end{abstract}

Key words: miniaturized MID-IR spectrometer, oil condition monitoring, pyroelectric line array, linear variable filter.

\section{Introduction}

Online oil condition monitoring may provide the possibility to change maintenance from fixed scheduled to wear dependent intervals. For machines in continuous operation or with limited access, for example stationary biogas engines or offshore wind turbines, online oil condition monitoring can contribute to increased production and significantly reduce maintenance costs. We recently demonstrated an infrared (IR) based analysis method providing accurate oil degradation data that show excellent agreement with laboratory based analysis results [1]. It has been shown that from the transmission spectra of the sample oil significant and application specific quantitative parameters such as acid number, oxidation, additive degradation and impurities can be derived.

\section{Materials and Methods \\ Infrared spectroscopy}

The underlying principle of the developed oil sensor is the absorption of infrared light by molecules. Molecules and functional groups absorb light at a specific unique wavelength by transforming the energy of the light into vibrational energy. Every type of molecule thus absorbs light at a specific wavelength and the intensity of the absorption is a measure of its concentration.

This principle is widely used in chemical laboratories all over the world what leads to extensive databases of used oil samples. The therefore used instrument is named FTIR Fourier transform infrared - spectrometer and is normally operated in a wavelength range of $4000 \mathrm{~cm}^{-1}$ to $500 \mathrm{~cm}^{-1}$ at a spectral resolution of $4 \mathrm{~cm}^{-1}$ or better.

\section{Miniaturized IR-spectrometers}

Our approach for a multi-purpose oil sensor is to use a miniaturized infrared spectrometer in a non-moving-part design (Fig. 1). 


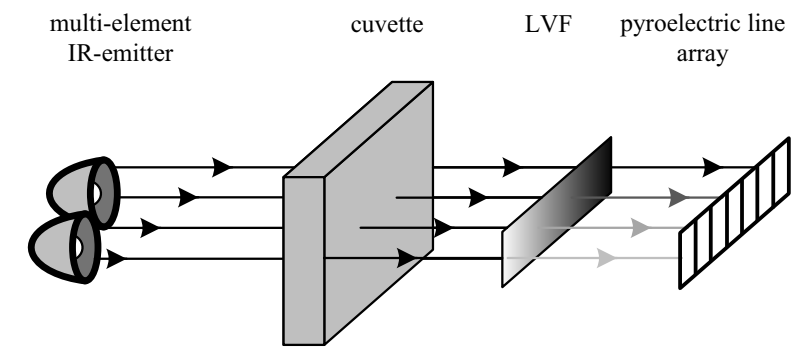

Fig. 1 LVF spectrometer featuring a linear detector array and fixed LVF atop. An electrical modulated IR emitter with parabolic reflector is used as infrared light source. Depending on the molecules and molecule chains in the oil probe, IR-radiation will be absorbed at different specific wavelengths. The LVF in front of the detector array selects a specific spectral band for each pixel.

In Fig. 1 a schematic drawing of the measurement systems is given. The main components are the infrared light source, a cuvette, a LVF, a linear pyroelectric detector array with application-specific integrated circuit (ASIC).

As electronics platform an embedded board is used to ensure a maximum on flexibility. So a stand-alone LVF-spectrometer without moving parts, well suited for condition monitoring tasks was developed.

As spectral apparatus a linear variable filter (LVF) is used. LVFs are small wedged FabryPérot etalons manufactured in a thin film optical coating process. The bottom layer is made of several Bragg reflectors forming a dielectric mirror. The next layer forms the cavity of the Fabry-Pérot resonator. Atop this resonator a second layer of Bragg reflectors is applied as upper dielectric mirror (Fig. 2).

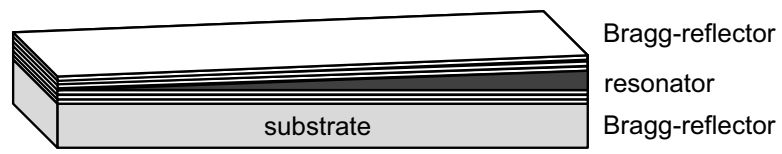

Fig. 2 structure of a linear variable filter (LVF).

Such filters normally are built on a suitable substrate material. In the MIR usually germanium substrates are used whereas in the VIS and VNIR the production process is compatible to fabricate it directly on a CMOS detector array [2]. The length of LVFs orientate on standard detector length of $12.8 \mathrm{~mm}$. The center wavelength $\lambda_{c}$ varies linearly over the filter length from $\lambda_{\min }$ to $\lambda_{\max }=2 \lambda_{\min }$. This $2: 1$ ratio is typical for LVFs and corresponds to the free spectral range (FSR) of Fabry-Pérot etalons. The spectral resolution $\Delta \lambda$ of a LVF is given by a constant factor $\mathrm{c}$ and follows:

$$
\Delta \lambda=\mathrm{c} \cdot \lambda_{\mathrm{c}},
$$

where $\Delta \lambda$ is the spectral resolution in $\mu \mathrm{m}, c$ is the FWHM of the LVF in \% of the center wavelength and $\lambda_{c}$ is the center wavelength of the respective bandpass which varies over the length of the filter. The used LVF has a $c$ of about $2 \%$.

Compared to FTIR-spectrometers the developed LVF-spectrometer has a reduced resolution and a limited spectral range. The used MIR type LVF operates in a range of about $1800 \mathrm{~cm}^{-1}$ to $900 \mathrm{~cm}^{-1}$ at a wavelength dependent resolution of about $36 \mathrm{~cm}^{-1}$ at $1800 \mathrm{~cm}^{-1}$ to $18 \mathrm{~cm}^{-1}$.at $1800 \mathrm{~cm}^{-1}$. In an earlier study the influence of reduced spectral resolution, limited spectral range and decreased signal-to-noise ratio (SNR) was investigated [3][4]. The analysis came to a clear result, that the main factor for accurate parameter quantification is the chosen spectral range. Here the MIR LVF was selected to fit this requirement. However the influence of the spectral resolution is rather low. It has even been observed that a reduced spectral resolution leads in some cases to a better quantification result because of the reduced spectral noise due to the smoothing effect of the LVF. But the system parameter SNR is affecting the quantification accuracy dominantly. The SNR is mainly influenced by the detector noise in our case a 128 pixel pyroelectric line array.

\section{Multivariata calibration}

The extraction of the oil quality parameters out of the measured infrared spectra requires sophisticated data processing. We use a multivariate regression model to predict the required oil quality parameters out of each measured infrared spectrum. As dependent variable information e.g. oxidation, TAN (total acid number) or additive condition, which was measured in a specialized, independent oil analysis laboratory is used. Firstly a learn data set was composed. This data set includes the infrared spectra $X$ as well as the dependent variable $Y$ which covers the entire range of the parameter level that will be predictable later. For each type of oil an individual model has to be built.

The prediction of the specific parameter levels is calculated using following equation:

$$
\hat{\mathbf{y}}=\mathbf{x}^{\mathrm{T}} \mathbf{b}+\mathrm{b}_{0},
$$


where $\hat{\mathrm{y}}$ is the predicted dependent variable, $x T$ is the measured infrared spectrum and $b_{0}$ is the static offset of the regression.

Two parameters are in particular relevant to measure the quality of the model. These are the Root Mean Square Error:

$$
\operatorname{RMSE}=\sqrt{\frac{\sum_{\mathrm{i}=1}^{\mathrm{n}}\left(\mathrm{y}_{\mathrm{i}}-\hat{\mathrm{y}}_{\mathrm{i}}\right)^{2}}{\mathrm{n}}}
$$

and the coefficient of determination:

$$
r^{2}=\sqrt{\frac{\sum_{i=1}^{n}\left(\hat{y}_{i}-\bar{y}_{i}\right)^{2}}{\sum_{i=1}^{n}\left(y_{i}-\bar{y}_{i}\right)^{2}}},
$$

where $\mathrm{y}_{\mathrm{i}}$ and $\hat{\mathrm{y}}_{\mathrm{i}}$ is the reference and the predicted parameter value, while $\overline{\mathrm{y}}_{\mathrm{i}}$ is the mean value of the reference parameter value. A good model is characterized by a low RMSE and $r^{2}$ close to one.

\section{Sample set}

To demonstrate the performance of the developed miniaturized LVF-spectrometer as oil condition sensor a representative set of samples spanning different types of oils and oil conditions was set up. In general oil degradation can be subdivided in the three interacting mechanisms base oil degradation, additive depletion and impurities. The oil quality parameters TAN (total acid number), TBN (total base number) and oxidation describe the process of base oil degradation which is mainly dominated by oxidation processes. Additive depletion describes the activation of the additives which are measured by the concentration of additive elements (e.g. molybdenum, calcium, magnesium, zinc, etc.) in the oil. Additives are mostly molecules with a central ion, so called non ash-free additives. Ash free additives can mainly be measured by the concentration of phosphorus.

If for example EP/AW-additives (extreme pressure / anti wear) exert their effect they deposit on the tooth flanks of the gears and consequently the additive concentration (indicated by the amount of its central ion in the laboratory ICP-analysis) is reduced. So a measurable explicit link between additive depletion, laboratory reference analysis (ICPOES, inductively coupled plasma- optical emission spectroscopy) and infrared spectroscopy is made. The third mechanism is the contamination which can be subdivided into internal and external causes. External causes are for example water, dust, or foreign oils due to a wrong refill. Internal causes are varnish, sludge or soot. Water and foreign oils can be directly quantified by their different absorption spectra and whereas soot for example is measurable through a signal attenuation of the infrared spectrum.

All of these three interacting mechanisms are directly measureable with one technique, the infrared absorption spectroscopy.

The quantification performance of the developed oil sensor is demonstrated by three example cases, covering all three oil aging mechanisms in the next section

\section{Results \& Discussion}

In this section the measurement and calibration results of the three selected case examples are shown. A direct comparison to quantification results based on a high resolution laboratory instrument (FTIR) is given.

\section{Base oil degradation}

Base oil degradation in engine oils is indicated by the oil quality parameters oxidation and TBN. In mineral oils oxidation is directly measurable by the infrared absorption band at $1710 \mathrm{~cm}^{-1}$. Fig. 3 shows 11 FTIR spectra of used oil in different stages of aging. The variation in the spectral plot contains information about the different aging mechanisms and in this case is visible with the naked eye.

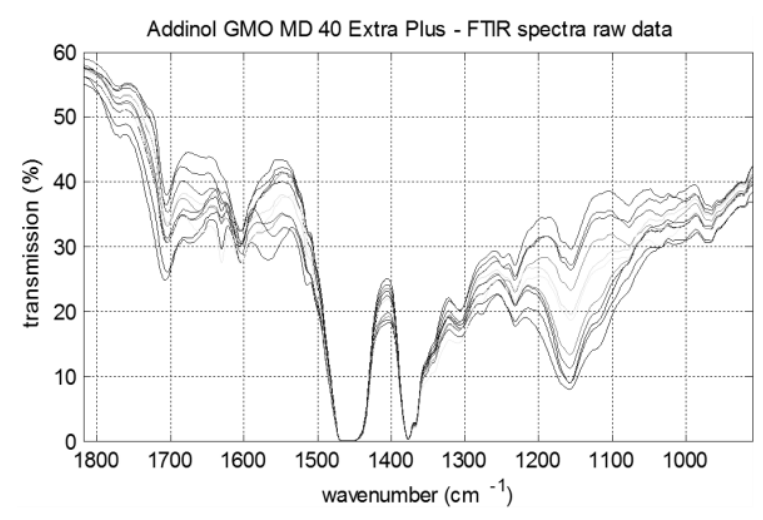

Fig. 311 in service oil samples of Addinol GMO MG 40 Extra Plus at different stages of aging measured with a FTIR-spectrometer at a resolution of $1 \mathrm{~cm}^{-1}$.

Despite the lower resolution of the developed LVF-spectrometer the variation in the spectral plot (Fig. 4) is also noticeable by inspection. The spectral features are smoothed but information retains and is still clearly and explicit usable by a multivariate quantification. 


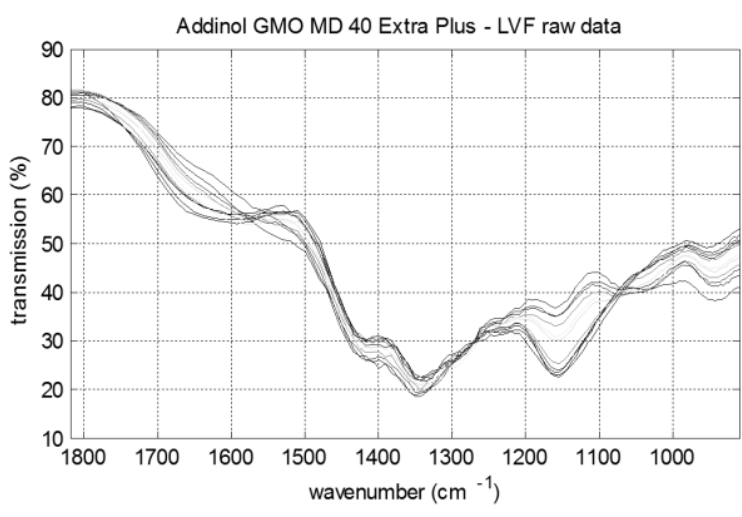

Fig. 411 in service oil samples of Addinol GMO MG 40 Extra Plus at different stages of aging measured with a LVF-spectrometer at a resolution of $2 \%$ FWHM.

A direct comparison of reference analytic and multivariate prediction based on spectral data can be visualized by the so called predicted versus measured plot. On the abscissa the results of the reference analytic and on the ordinate the prediction results of the multivariate calibration is plotted. Are both quantifications identical all samples would lie on a line through the origin with a slope of one (indicated as a black line in the plots). The quality of the model is hereinafter described by RMSE (see equation 3 ) and $r^{2}$ (see equation 4 ).

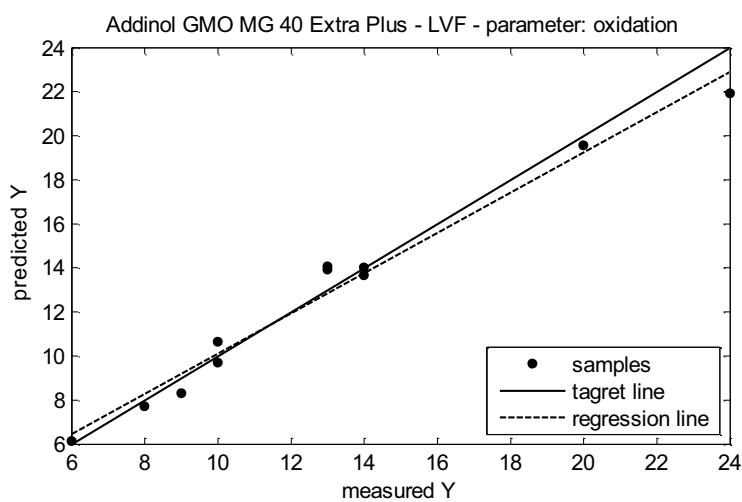

Fig. 5 Predicted versus measured plot of 11 in service oil samples of Addinol GMO MG 40 Extra Plus at different stages of aging measured with a LVF-spectrometer (predicted) and with a FTIRspectrometer (measured).

Quantification results of the oil quality parameter oxidation for Addinol GMO MG 40 Extra Plus are given in Tab. 1.

The predicted versus measured plot for quantification of the TBN is shown in Fig. 6. As a reference analytic the results of a potentiometric titration is used. Quantification results are summarized in Tab. 1.

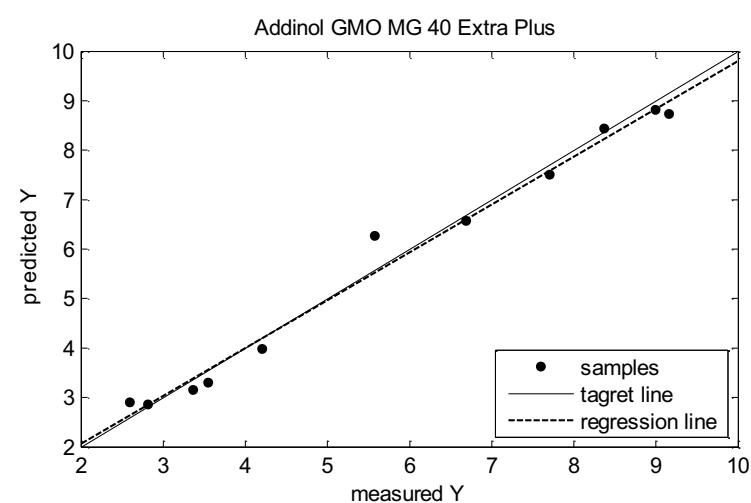

Fig. 6 Predicted versus measured plot of 11 in service oil samples of Addinol GMO MG 40 Extra Plus at different stages of aging measured with a LVF-spectrometer (predicted) and with potentiometric titration (measured).

Tab. 1 Comparison of prediction qualities for oil type Addinol GMO MG 40 Extra Plus.

\begin{tabular}{|c|c|c|c|}
\hline parameter & instrument & $\mathbf{r}^{\mathbf{2}}$ & RMSE \\
\hline \multirow{2}{*}{$\begin{array}{c}\text { Oxidation } \\
(\mathrm{A} / \mathrm{cm})\end{array}$} & LVF & 0.972 & 0.838 \\
\cline { 2 - 4 } & FTIR & 0.930 & 1.330 \\
\hline \multirow{2}{*}{$\begin{array}{c}\text { TBN } \\
(\mathrm{mgKOH} / \mathrm{g})\end{array}$} & LVF & 0.984 & 0.304 \\
\cline { 2 - 4 } & FTIR & 0.984 & 0.309 \\
\hline
\end{tabular}

For both significant oil aging parameters of this oil type, a reliable prediction was achieved with the developed oil sensor. Values for $r^{2}$ are at FTIR level (TBN) as well as the RMSE. For the Oxidation the LVF-spectra provide even better results in terms of $r^{2}$ and RMSE.

\section{Additive depletion}

A further important parameter giving the oil quality is the amount of remaining additives in the oil. This parameter is the first to change when oil is aging.

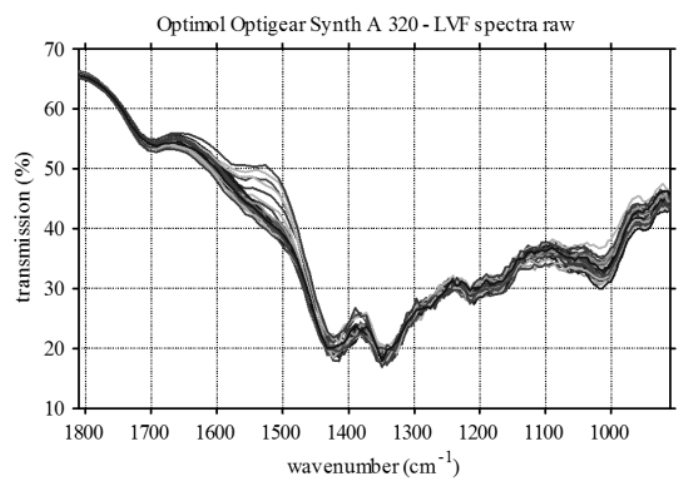

Fig. 750 in service oil samples of Optimol Optigear synth A 320 at different stages of aging measured with a LVF-spectrometer at a resolution of $2 \%$ FWHM.

The additivation of oils is strongly changing from type to type and the exact formulation is kept secret by the oil companies. Nevertheless 
additive depletion can be measured by their amount of additive elements. This amount is determined by atomic emission spectroscopy in this case an inductively coupled plasma (ICP) mass spectrometer.

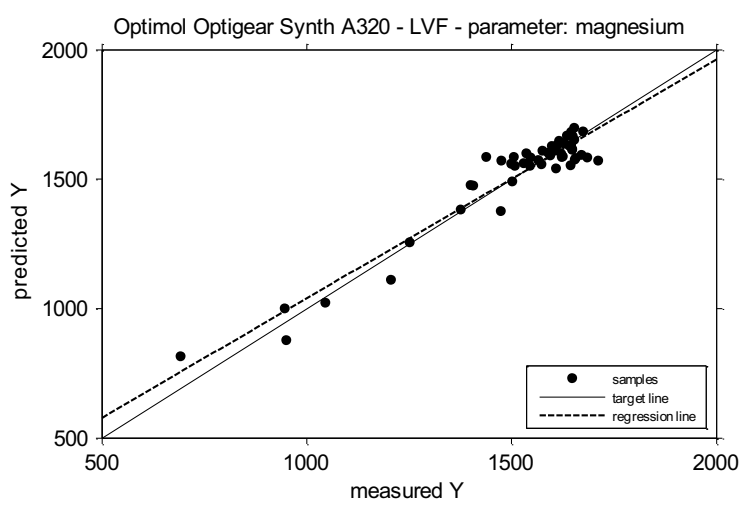

Fig. 8 Predicted versus measured plot of 50 in service oil samples of Optimol Optigear synth A 320 at different stages of aging measured with a LVFspectrometer (predicted) and with ICP (measured).

The values of the reference analysis and the prediction based on LVF-spectra are exemplarily compared for the elements magnesium (see Fig. 8) and phosphorus (see Fig. 9 ).

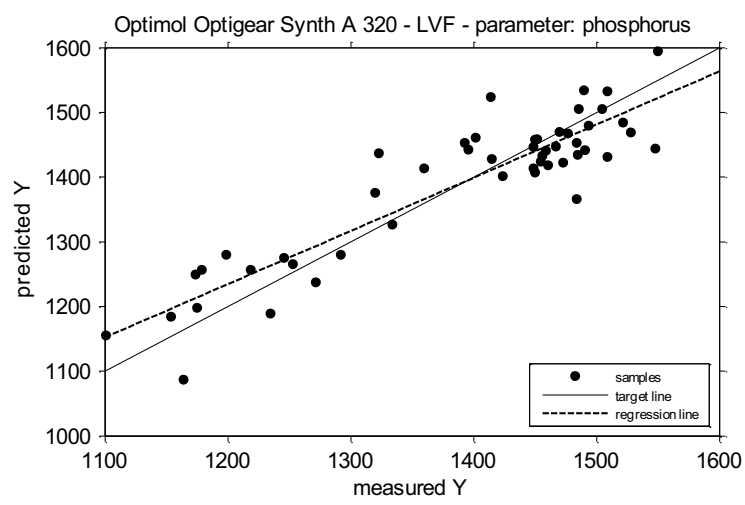

Fig. 9 Predicted versus measured plot of 50 in service oil samples of Optimol Optigear synth A 320 at different stages of aging measured with a LVFspectrometer (predicted) and with ICP (measured).

Tab. 2 Comparison of prediction qualities for oil type Optimol Optigear synth A 320.

\begin{tabular}{|c|c|c|c|}
\hline parameter & instrument & $\mathbf{r}^{\mathbf{2}}$ & RMSE \\
\hline $\begin{array}{c}\text { Magnesium } \\
\text { (ppm) }\end{array}$ & LVF & 0.920 & 59.532 \\
\cline { 2 - 4 } & FTIR & 0.916 & 60.823 \\
\hline $\begin{array}{c}\text { Molybdenum } \\
\text { (ppm) }\end{array}$ & LVF & 0.923 & 81.704 \\
\cline { 2 - 4 } & FTIR & 0.933 & 76.392 \\
\hline $\begin{array}{c}\text { Phosphorus } \\
\text { (ppm) }\end{array}$ & LVF & 0.826 & 51.335 \\
\cline { 2 - 4 } & FTIR & 0.773 & 58.530 \\
\hline
\end{tabular}

For all three significant oil aging parameters concerning the additive depletion of this type of oil, a reliable prediction was achieved with the developed oil sensor. Values for $r^{2}$ are comparable to FTIR levels, sometimes even slightly better, what in this case might be lead back to the uneven distribution of the samples. Also the RMSE values are in the same range. This example case shows a high performance of the developed oil sensor for detecting additive depletion.

\section{Impurities in the oil}

One of the most important contaminants to measure is water. High water concentrations will lead to serious oil degradation. Ester based oils will suffer from hydrolysis and also frequently used additives like zincdithiophosphate or calcium-sulfonate will be decomposed faster. To evaluate the ability of the developed miniaturized LVF-spectrometer to measure the water content in oil, a practically relevant sample set was prepared. This sample set contains concentrations from 250ppm up to $5000 p p m$ and was mixed into fresh KlüberSynth GEM4-320N.

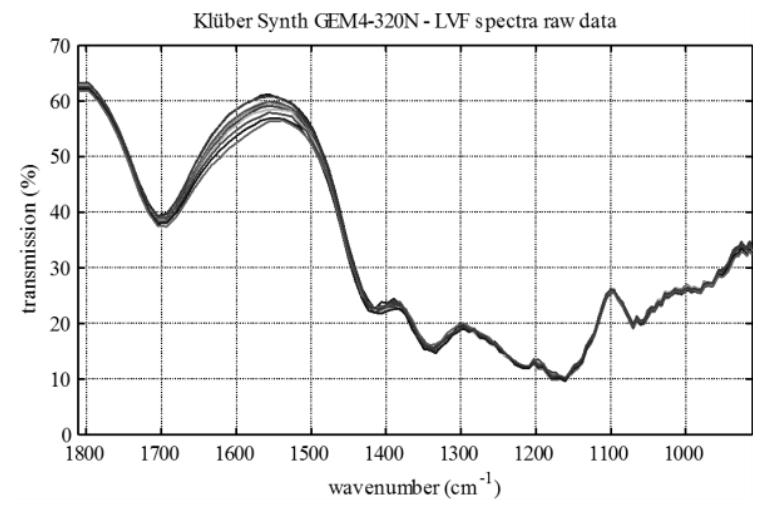

Fig. 10 Spectra of 10 oil samples having different concentrations of water in the range from $250 \mathrm{ppm}$ up to 5000 ppm.

The raw transmission spectra of the 10 oil samples are shown in Fig. 10. In the spectral range around $1645 \mathrm{~cm}^{-1}$ the absorption band of the $\mathrm{H}_{2} \mathrm{O}$ molecule ( $\mathrm{v}_{2}$ bending vibration) can be measured. The different concentrations lead to different absorptions in this range what can be directly noticed in Fig. 10 and used to quantify the water concentration.

A multivariate calibration for the determination of the water content was performed using the unmodified raw data of the measurements. The spectra were averaged over 500 measurements what gives a total measurement time of 160 seconds. Also spectra taken by an averaged measurement of 100 , respectively 30 seconds delivers good results. Since in oil condition monitoring tasks the measurement time is not a 
limiting factor we decided to use the higher averaging in this case.

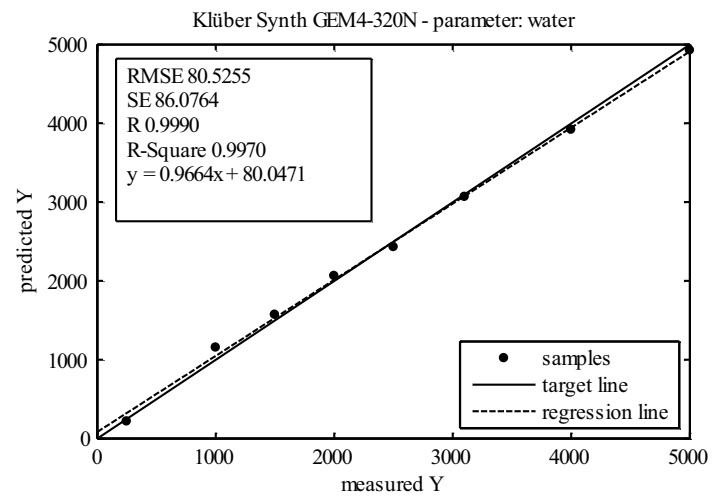

Fig. 11 Predicted vs. measured plot of 8 oil samples of different water concentrations from 250ppm up to 5000ppm.

Alert levels for this type of oil are between 2000 ppm to 3000 ppm with a recommendation to change the oil at concentrations higher than $3000 \mathrm{ppm}$. The achieved accuracy of predicting water with the miniaturized IR-spectrometer is about $80 \mathrm{ppm}$. This mean deviation between the reference analysis according to the Karl-Fischer method and the miniaturized IR-spectrometer is very low and lies certain magnitudes under the critical alert level of $3000 \mathrm{ppm}$. The high accuracy of the model is also supported by a $r^{2}$ of 0.997 and a very steep regression line with a slope of 0.966 .

\section{Conclusion \& Outlook}

In this paper a novel multi parameter oil condition sensor was demonstrated. The sensor is based on a rugged miniaturized infrared spectrometer without moving parts. This sensor brings a well known laboratory method - infrared spectroscopy - to the field and provides online oil condition data. The accuracy of predicted oil quality from the measured spectra was demonstrated for the three interacting oil aging mechanisms base oil degradation, additive depletion and impurities. All three mechanisms could be quantified in good quality giving well known values like oxidation, TAN, TBN, additive elements and water concentrations. All crucial oil quality parameters can be determined in more than sufficient reliability. The developed oil sensor can be used for nearly all types of oil and needs just a single calibration for it. Also initially not modelled parameters can be interpreted later using the saved spectra. Combining the measured oil quality parameters over time with simultaneously captured machine parameters makes maintenance planable. Also machine health condition could be accurately evaluated and break downs with all their consequences could be successfully prevented. The developed LVF-spectrometer could also be used for many further analytes and applications.

\section{Acknowledgement}

This work was supported by a research grant of the Bayrisches Staatsministerium für Wirtschaft, Infrastruktur, Verkehr und Technologie coordinated and supervised by VDI/VDE. We thank Oelcheck $\mathrm{GmbH}$ and Klueber Lubrication Munich SE \& Co. KG for their support and the many helpful discussions. We also want to thank TUM graduate school for their support.

\section{List of references}

[1] B. R. Wiesent, D. G. Dorigo, and A. W. Koch, "Miniaturisierte Infrarot-Spektrometer zur Ölzustandsüberwachung in OffshoreWindkraftgetrieben," Technisches Messen, vol. 79, no. 1, pp. 1-8, 2012.

[2] A. Emadi et al., "Fabrication and characterization of IC-Compatible Linear Variable Optical Filters with application in a micro-spectrometer," Sensors and Actuators A: Physical, vol. 162 , no. 2, pp. 400-405, Aug. 2010.

[3] B. R. Wiesent, D. G. Dorigo, M. Schardt, and A. W. Koch, "Gear oil condition monitoring for offshore wind turbines using band limited low resolution spectra," in Proceedings of Oildoc 2011, 2011.

[4] B. R. Wiesent, D. G. Dorigo, and A. W. Koch, "Suitability of tunable Fabry-Perot spectrometers for condition monitoring purposes of gear oils in offshore wind turbines," in Proceedings of 12th International Conference on Infrared Sensors \& Systems, 2011.

[5] H. Abdi, "Partial least squares regression and projection on latent structure regression (PLS Regression)," Wiley Interdisciplinary Reviews: Computational Statistics, vol. 2, no. 1, pp. 97-106, Jan. 2010. 